\title{
A Hybrid Leaf Disease Detection Scheme Using Grayco-Occurance Matrix Support Vector Machine Algorithm
}

\author{
K. Subhadra, N. Kavitha
}

\begin{abstract}
An Indian economy depends upon the agriculture up to $70 \%$ approximately. Hence, there is a need to Take care of agriculture and its resources. In such aspects, the plant disease and leaf disease is one of the major concerns that affect the overall processing of producing food, feed, fiber and many other favorite products by the cultivation. It is one of the reasons that disease identification and detection in plant adopts a significant job in agro industry area. Due to this reason, appropriate detection methodology consideration is to be taken here. Most of the research focused more on combining image processing and soft computing algorithms to solve this issue. With this motivation, this research utilize Median filter for noise removal in initial stage. Later, Hue-Saturation-Value is used for preprocessing. Further, Fuzzy C-Means Clustering (FCM) considered for clustering image samples at different iteration. Finally, the research considered a hybrid mechanism by combining Gray Co-Occurrence Matrix and Support Vector Machine. Further, the proposed method results better outcome in terms of efficiency as $87.43 \%$ K-nearest neighbor (KNN) classifier, Color Transform and Exponential Spider Monkey Optimization.
\end{abstract}

Keywords---Leaf Disease Detection, Gray Co-Occurrence Matrix, Support Vector Machine, K-Nearest Neighbor Classifier, and Color Transform, Exponential Spider Monkey Optimization.

\section{INTRODUCTION}

Generally, plants and trees have spots, taints and some other diseases in their leaves. This issue turn into serious issue once it cross some extent due to pollution, environment changes, and industrial disturbances and so on. Finally, the quality and amount of horticultural items will be degraded. A larger part of the developing national populace relies upon horticulture yields. Farmers have wide scope of decent variety to choose reasonable natural product or vegetable harvests to develop. Since, the development of these harvests for perfect profit and excellence outcome is profoundly specialized and testing. It tends to be improved by the guide of pioneering assistance and automated cultivating. The administration of perpetual harvests requires nonstop and close observing particularly for the administration of illnesses that can influence creation altogether.

Numerous researchers have fragmented the upgrading techniques for the programmed identification and grouping of leaf ailments dependent on most important goals like spectral and stereo image formats. Likewise, most of the recent image processing techniques, data mining and artificial intelligence results in better result. However, the major task of such process is depending upon the input

Manuscript received September 16, 2019.

K. Subhadra, Research Scholar, Nehru Arts and Science College, Coimbatore. T.N, India. (e-mail: subha.badra@gmail.com)

Dr.N. Kavitha, HOD, Associate Professor, Nehru arts and Science College, Coimbatore. T.N, India. dataset. The fragmenting algorithm should be capable of handling complex dataset.

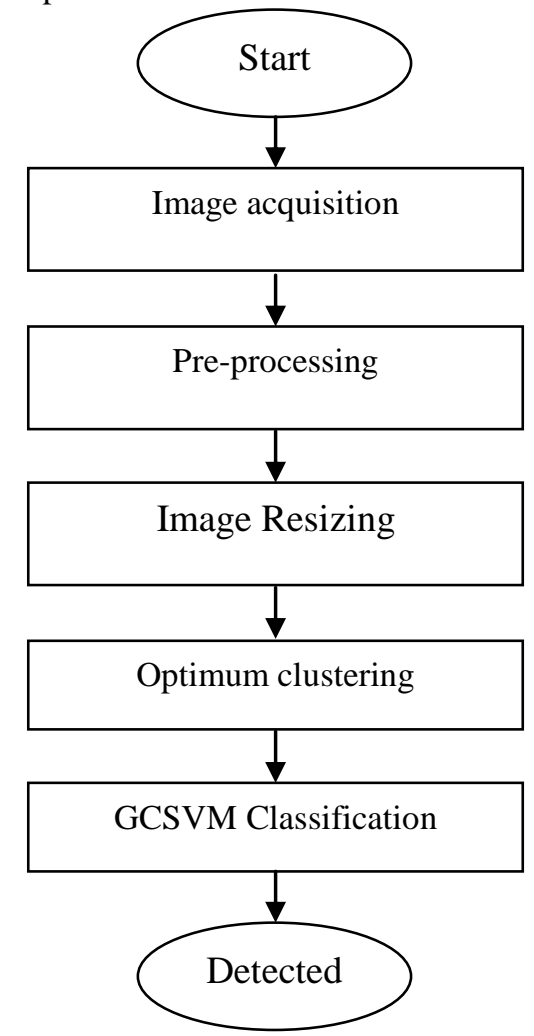

Figure 1: Flow chart for the research

Figure 1 discusses about the concept of image processing. Initially, this research is framed for image detection and later it will modify into automated prototype detection model. Initially, the image is processed with the median filter and resized is to be happen. Next, the optimized clustering is to be processed with the Fuzzy $\mathrm{C}$ means clustering. At later stage, classification is to be carried out with the hybrid model named as Gray Co-Occurrence Matrix-Support Vector Machine (GCSVM) Algorithm. It includes SVM classifier because it has a multi objective function for fitness.

Pre-processing- Generally, pre-processing mainly used to enhance and process the acquisition image. It is an significant step in the overall detection process because for a noisy image, has a direct impact on the end result of plant identification. The pre-processing stage performs two tasks, namely, de-noising and segmentation.

De-noising-The method enhances an input leaf image in three steps, namely, de-noising, contrast adjustment and edge enhancement.

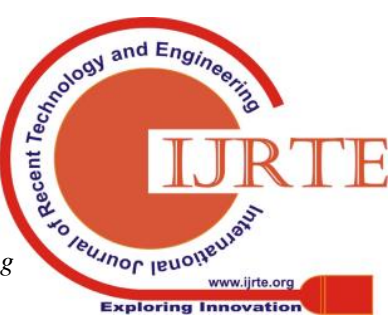




\section{A HYBRID LEAF DISEASE DETECTION SCHEME USING GRAYCO-OCCURANCE MATRIX SUPPORT VECTOR MACHINE ALGORITHM}

Most of the de-noising solutions focuses on noise removal and ignore the edge and contrast details. Some methods apply separate algorithms for each of these three steps.

Segmentation- To regulate the division system, previous steps including picture resizing (Conversion to $512 \times 512$ pixel estimate), Red Green Blue (RGB) to gray scale transformation, leaf external layer evacuation and plant detail expulsion are finished. Change of picture power or shading guide esteems is performed to deal with medicinal data misfortunes stimulated because of picture resizing. Efficient and effective image segmentation is important for accurate plant identification through leaf recognition. The technique proposed in this phase focus on separating the Region of Interest (ROI), from its contextual. Among the various methods used, wavelet based approaches was more suitable for segmenting images from its background. But due to the varied color and texture property of leaf images, wavelets have the shortcoming of grouping regions that result in inaccurate segmentation. Therefore to resolve this problematic, texture, color or a fusion of these features were used for grouping regions. The algorithm exploits color and texture features in wavelet domain and uses Fuzzy $\mathrm{C}$ means grouping calculation during segmentation.

Feature Extraction and Selection-The principle point of the subsequent stage is to change over the picture information into an arrangement that streamlines the way toward coordinating between leaf pictures. This stage comprises of two stages, in particular, Feature Extraction and Feature Selection. The element extraction step capacities to find different highlights that best speak to a leaf picture. As the quantity of highlights chose is ordinarily extremely high, a component determination calculation is utilized in the subsequent stage, to choose the most unmistakable highlights. During highlight extraction, five classifications of highlights were removed. They are geometric features, color features, texture features, fractal features and leaf related features.

Classification- The last period of the examination is the assignment of distinguishing the plant to which the info leaf has a place and is significant for herbal field. The calculation for the most part rotates cycle an iterative acknowledgment methodology that matches the highlights extricated from the information picture with highlight vectors speaking to the leaf pictures of the pre-fabricated. Primarily, Gray Co-Occurrence Matrix (GCM) is implemented with a machine learning algorithm such as SVM classifier is considered for this process. The field of leaf malady identification for plant grouping has encountered an expanded requirement for quick and effective characterization calculations that guide in monitoring significant tea plants.

The tea plant infection distinguishing proof through leaf acknowledgment is an exploration field that has caught the consideration of numerous botanists for quite a while. Only during the last few years, the advantages of using computers to perform plant identification using leaf have been predicted.

Most of the current researches use neural networks (Sladojevic et al., 2016), wavelets (Harini and Bhaskari, 2011) and fuzzy network (Rastogi et al., 2015) for automating the classification process. These systems are applicable only to explicit species and regularly require Human arbitration to characterize terms for highlight extraction and pre-handling. The efficiency of these systems thus depends on the expertise of knowledgeable experts.

The motivation of the research is taken from the following factors. Naked eye perception by specialists is the most obvious and existing strategy for plant disease identification and location.

Since, the previous strategies are advantageous just when farms are smaller in size. With respect to the bigger farms this strategy is very complex. A major group of specialists and their continuous research is required for doing as such which results in surprising expense as far as both time and work.

In the majority of the countries the farmers can't find the exact solution for plant diseases and techniques to anticipate them or manage them. They are not ready to contact the specialists in time for their recommendation and if they do as such it is again tedious and costly procedure.

Hence, to avoid such complexity this research focused more on detecting the exact problem that existing in leaf. This investigation mainly emphases on a spontaneous system that helps to develop and detect functional and irrational features in plant leave. The prototype model is developed with its application to judge leaf related diseases. Therefore, the basic objectives of the research are:

i. Image acquisition and pre-processing, characterization of infected tea plant leaves.

ii. Screening different varieties from various hills and analysed with various parametric.

iii. Initially, filter the noise by median filter and further processed with the FCM Clustering.

iv. Further, the unit is hybridized with the Gray CoOccurrence Matrix and Support Vector Machine (SVM).

v. At last, to verify the exact formulation of proposed hybrid concept some existing methodologies likeKnearest neighbor (KNN) classifier (Hossain et al., 2019), Color Transform (Chaudhary et al., 2012) and Exponential Spider Monkey Optimization (Kumar et al., 2018).

Further, this research is prearranged as mentioned below: Section II labels about various research activities with recent years.

Section III describes about the research methodology with hybrid combination. Section IV describes about the experimental results and its outcome. Finally, section V summarizes the entire concept.

\section{LITERATURE SURVEY}

Tea, being a monetarily advantageous yield, is regularly compelled to develop under differing climatic and soil conditions remote from its regular habitat.

The benefit related with tea development has likewise made it a subject of different social medicines which are generally in change with its normal conditions for development. 
Tea is an estate crop which requires the developing of the enduring plant in a pure stand reaching out over a time of around 40-100 years and over tremendous regions which bears a glad chasing ground for vermin and infections of various types. In this research, the tea leaves are considered but literature will follows some other leaf detection methods and implementations.

Al Bashish et al., (2011) proposed a concept of K-means based segmentation and neural network based classification. It is framed to support various automatic features that should follow cheap and efficient.

The overall features are extracted with texture with some training data set. Stereomicroscopic strategy and Image investigation technique is thought about for value of picture examination as a proficient and exact strategy to gauge natural product qualities like scope, figure dispersion associated structures by Mix et al., (2003).

Overall image length got with image investigation was fundamentally more remarkable than that recorded with a stereomicroscopic. Just organic product length assessments did not vary between the two techniques.

All things considered there was an exceedingly noteworthy connection between organic product length appraisals acquired from the two strategies for all types of study. This demonstrates both stereomicroscopic and picture investigation precisely separated products of various sizes. It was presumed that image investigation has following points of interest:

- The high measure of organic product parameters acquired with unique estimation

- It is very simple to manipulate human faults

- The expected period to acquire enormous informational collections are reduced greatly by concerning organic product quality changeability characteristics of organic products with muddled shapes.

Pest leaves unmistakable outward consequences for florae like rolling the greeneries or wrecking the entire plant. The sucking nuisance diminishes the dampness substance of the leaves. Every one of these impacts changes the chlorophyll substance of a plant with relating variety in its unearthly picture.

Abdullah and Umer (2004) talked about the conceivable outcomes for identifying these impacts by utilizing different remote detecting procedures for procurement of ghostly picture by satellite symbolism, airborne pictures from sanctioned or model planes. An epic methodology is proposed by Ei-Helly et al., (2004) for coordinating picture examination strategy into symptomatic master framework. A Central Laboratory of Agricultural Expert System (CLASE) symptomatic model is utilized to oversee cucumber crop. The master framework discovers the sicknesses of client perception.

So as to analyse a complaint from a leaf picture, different image preparing stages are utilized: upgrade, division, include extraction and arrangement. They tried three unique issues, for example, Leaf excavator, Powdery and Downey.

The proposed methodology has incredibly diminished blunder inclined exchange among framework and client. The morphological highlights of leaves are utilized for plant
- The likelihood to assess changeability in

order and in the early analysis some exact diseases. Tzionas et al., (2005) presents structure and usage of a counterfeit vision framework which concentrates explicit regular highlights from plant leaves.

The proposed framework comprises of a counterfeit vision framework (camera), a blend of picture preparing calculations and feed forward neural system.

An effective Computer-Aided Plant Species Identification (CAPSI) approach is proposed by Du et al., (2006), which depend on plant leaf pictures utilizing a shape coordinating system. Right off the bat, a Douglas - Peucker guess calculation is received to the first leaf shapes and another shape portrayal is utilized to frame the arrangement of invariant traits. At that point a Modified Dynamic Programming (MDP) calculation for shape coordinating is proposed for the plant leaf acknowledgment.

At long last, the predominance of our proposed strategy over customary ways to deal with plant species distinguishing proof is shown by investigation.

The trial result demonstrated that our proposed calculation for leaf shape coordinating is truly reasonable for the acknowledgment of unblemished as well as incomplete, mutilated and covered plant leaves because of its robustness.

The multi-scale distance matrix network is utilized for maintaining the shape geometry while being invariant to interpretation, turn, scaling, and reciprocal symmetry, Hu et al (2012). The descriptor is additionally joined with a dimensionality decrease to recover its discriminative power. The proposed strategy gaps the tedious point savvy coordinating experienced in the vast majority of the recently utilized shape acknowledgment calculations. It is in this manner quick and reasonable for continuous applications.

They connected the proposed technique to the task of plan leaf acknowledgment with examinations on two informational indexes, the Swedish Leaf informational index and the Intelligent Computing Laboratory (ICL) Leaf features collection. The trial results unmistakably show the viability and productivity of the proposed descriptor.

Prasad et al (2011) considered Relative Sub-image coefficients (RSC) features extracted from leaf images and is used for classification and used SVM classifier to execute a mechanized leaf acknowledgment framework for plant leaf distinguishing proof and order.

Programmed plant species distinguishing proof and grouping is useful in science, timberland and horticulture to think about and find new species in plant in nurseries and is also used to see the remedial plants to prepare home developed prescriptions.

Here, 300 leaf features are removed from a single leaf of 624 leaf dataset to mastermind 23 different sorts of plant species with an ordinary precision of $95 \%$.

Differentiated and various approachs, our proposed count has less time multifaceted nature and is definitely not hard to use with higher accuracy.

Current examination used the picture dealing with systems to portrayal of plants subject to leaves affirmation. 


\section{A HYBRID LEAF DISEASE DETECTION SCHEME USING GRAYCO-OCCURANCE MATRIX SUPPORT VECTOR MACHINE ALGORITHM}

Two systems called the Gray-Level Co-occasion Matrix (GLCM) and Principal Component Analysis figurings have been associated by Ehsanirad and Kumar (2010) to remove the leaves surface features. To amass 13 sorts of plants with 65 new or deformed leaves as test pictures, the Algorithms are set up by 390 leaves. The outcomes abridged that the exactness of PCA system with $98 \%$ end up being greater profitability stand out from the Gray-Level Co-Occurrence Matrix (GLCM) procedure with $78 \%$ accuracy.

Sladojevic et al., (2016) depicted the most recent adaptation of Convolutional Neural Networks (CNNs) for tea picture arrangement. It talks about the model of plant maladies recognition model, in light of leaf picture portrayal, by the use of significant convolutional systems. Novel technique for getting ready and the philosophy used support an unexpected and basic system execution by and by.

The created model can see 13 particular sorts of plant sicknesses out of strong leaves, with the ability to perceive plant leaves from their condition. As demonstrated by our knowledge, this procedure for plant disease affirmation has been proposed out of nowhere.

Each and every major development required for executing this sickness affirmation model is totally delineated all through the paper, starting from social event pictures in order to make a database, assessed by country masters. Caffe is a profound learning system, initially created at University of California, made by Berkley Vision and Learning Center, was used to play out the significant CNN getting ready. The exploratory results on the made model achieved exactness some place in the scope of $91 \%$ and $98 \%$, for free class tests, all around $96.3 \%$.

Further, some other researchers are focused more on discussing about the leaf detection concept such as laseractuated breakdown spectroscopy joined with the discriminant investigation technique (Wang et al., 2016), support vector machine (Hossain et al., 2018), Simple Linear Iterative Cluster (SLIC) with SVM (Sun et al., 2019) and so on. With the motivation of each research, this research is focused more on tea leaves diseases detection with each hybrid method by utilizing super vector machine .

Color Transform Based Approach for Disease Spot (Chaudhary et al., 2012) utilized the concept of flowering plants leaf detection and angiosperms type plant. Based on the concept, the proposed method consist of three types of comparisons such as CIELAB, Hue, Saturation, Intensity (HIS) and $\mathrm{Y}^{\prime}$ is the luma component and $\mathrm{CB}$ and $\mathrm{CR}$ are the blue-difference and red-difference chroma components $(\mathrm{YCbCr})$ color space were considered for the process of disease spot detection.

They considered Median filter for image smoothing. Finally threshold can be calculated by applying Nobuyuki Otsu.

The concept of utilizing this method is because of its automatic performs clustering-based image thresholding. The advantage of this method is reduction of a gray-level image to a binary image. Otsu method is considered on color component to detect the disease spot.

Hossain et al., (2019) actualized the idea of KNN classifier. It includes two phases. One is getting ready stage where the leaf pictures are set apart with their classes what's

increasingly, another is attempting stage where the leaf pictures are unlabeled and count yields the once-over of $\mathrm{k}$ nearest data point (planning data point) to stamp the unlabeled point and gatherings their classes. KNN rules are: The plan of set away planning and testing data.

Use euclidian partition which can speak to by division parameter to check the detachment between set away records and darken record to arrange. It is spoken to by the $\mathrm{K}$ closest neighbors and uses class names of closest neighbors to discover the class mark of obscure information by tallying greatest vote.K-NN classifier algorithmic steps

- Initialize the data and Initialise the estimation of $\mathrm{k}$

- For getting the foreseen class, accentuate from 1 to signify number of planning data centers

- Calculate the detachment between test data and every section of getting ready data. Here we will use Euclidean detachment as our division metric since it's the most notable strategy. Various estimations that can be used are Chebyshev, cosine, etc.

- Sort the decided partitions in climbing demand subject to detachment regards

- Get top k lines from the masterminded group

- Get the most ceaseless class of these lines

- Return the foreseen class

Kumar et al., (2018) presents a novel exponential spider crwly monkey progression which is used to fix the basic features from high dimensional course of action of features made by SPAM.

Moreover, the picked features are reinforced to enable vector to machine for course of action of plants into debilitated plants and strong plants using some noteworthy characteristics of the leaves.

The exploratory outcomes outline that the picked features by Exponential SMO effectively increase the plan unfaltering nature of the classifier interestingly with the thought about component decision systems.

- An epic exponential Spider Monkey Optimization (ESMO) methodology has been exhibited.

- The extraction of relevant features from the considered leaf pictures done using SPAM.

- A new philosophy for assurance of feature subset has been anticipated reliant on ESMO.

- For gathering the sound leaf pictures and undesirable leaf pictures, kNN, SVM, ZeroR, and LDA classifiers are penniless down.

The SMO is correspondingly new count reliant on the logical model of shrewd direct of bug monkeys that seek after the parting combination social structure (FFSS).

According to FFSS, 100 monkeys appropriate themselves from more prominent to minor social events and the other path around for scrounging.

The rule characteristics of the FFSS are according to the accompanying:

- Initially, all creepy crawly monkeys continue on in the get-togethers of 40-50 individuals. Each basic social affair has a pioneer under whom the sustenance sources are explored.

- It is named as an overall pioneer of that social occasion. 
- In example of insignificant measure of sustenance, the overall pioneer make smaller subgroups from greater social event with each subgroup containing three to eight people to search independently and each subgroup headed by neighborhood pioneer.

- The choice of looking through nourishment in each sub-bunch is additionally chosen by a pioneer, known as nearby pioneer.

- The bunch individuals keep up social security's and guarded limits by conveying among themselves and with different individuals from the gathering utilizing a special sound.

\section{PROPOSED METHODOLOGY}

The proposed automatic leaf detection unit is framed based on the following aspects. Initially, the image is acquired by camera with various agricultural fields. Specifically, this research is focussed fully on tea leaves diseases detection. In future, we can change the leaf and measure the accuracy and detection. After acquisitions, the image is processed through the pre-processing unit. Here, Median filter is considered to process the image without noise. Since, the retrieved image may contain noise. Hence, median filter deployed before pre-processing stage.

The middle ' $\mathrm{m}$ ' of a ton of regards is the worth that has the property that an enormous segment of the characteristics in the set are not actually ' $\mathrm{m}$ ' and half are more unmistakable than ' $m$ '. Middle separating is the action that replaces each pixel by the center of the diminish measurement in the zone of that pixel. Center channels are non-straight directs in light of the way that for two groupings $x(n)$ and $y(n)$.

For the most part, these sorts of channel are useful in removing detached lines or centers (pixels) while ensuring spatial objectives. They perform very well on pictures containing parallel (salt and pepper) racket yet perform inadequately when the confusion is Gaussian. Their show is similarly poor when the amount of upheaval pixels in the window is more critical than or an enormous bit of the amount of pixels in the window.

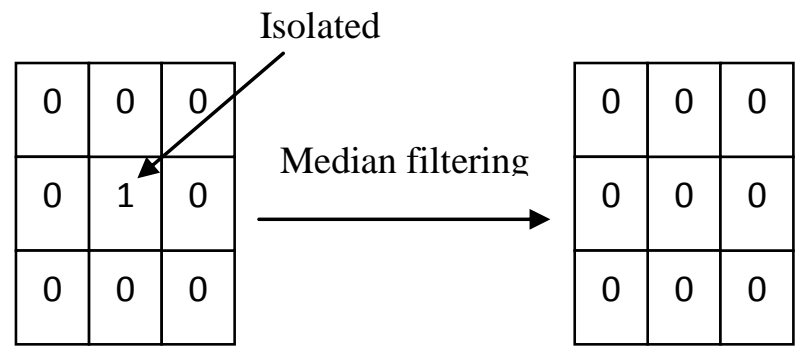

Figure 2: Median Filtering

This exploration used tint, immersion, esteem (HSV) based shading model. Since, it incredibly diminishes the size of shading and dim scale data of a picture. A lot of detached focuses are bunched into articles by shading extraction. Hue filtering is utilized to fragment the predefined shading. Shade separating is utilized to section the predetermined shading. The hues on the furthest edge of the shading circle are the "tones", which are hues in their most flawless structure. This procedure can keep filling in hues around the wheel. The following level hues, the tertiary hues, are those hues between the optional and essential hues

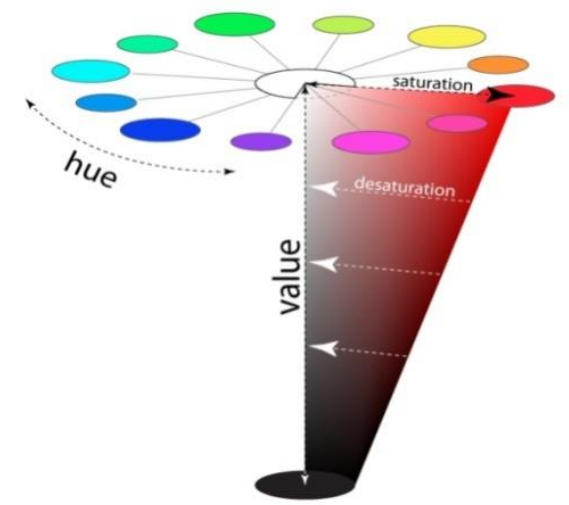

Figure 3: Hue, Saturation and Value

Saturation is likewise alluded to as "intensity" and "chroma." It alludes to the predominance of tone in the shading. On the external edge of the shade wheel are the 'pure' hues. If we concentrate on focal point of the wheel, the tone which is utilizing to depict the shading rules less and less. When you achieve the focal point of the wheel, no shade overwhelms. These hues straightforwardly on the focal pivot are considered de-saturated. At present, the "value "is considered for HSV scale. Value is the component of gentility/murkiness. As far as shading, esteem portrays the general force or quality of the light. On the off chance that tone can be thought of as a measurement bypassing a wheel, at that point worth is a direct pivot going through the center of the wheel, as observed below:

Presently, on the off chance that you visualize that each shade was likewise spoken to as a cut like the one above, we would have a strong, upside down cone of tints. The model spoke to in figure 3 saw as a cut of the cone. Notice, how the right-most edge of this cone cut exhibits the best proportion of the other contending tones, and how as you go down vertically, it gets darker in "regard." Also consider that to be we adventure out from proper to left in the cone, the shade ends up being less overarching and over the long haul ends up being absolutely de-soaked along the vertical focal point of the cone. This vertical center of complete desaturation is implied as dim scale. At the point when the estimation of shade is set, a veil is connected to the picture. In a twofold picture, when the estimation of pixels fulfils a predefined foundation, for example, tint, the worth changed by covering. Capacity is set to zero which shows in white shading.

In the given picture a preservationist FCM calculation does not completely utilize the spatial data. In this paper, we present FCM which joins the extraordinary data in to a participation capacity utilized for bunching. Here the uncommon capacity is the summation of the given enrolment capacity of the area of the every pixel. The main objective of selecting FCM is because it has homogeneity of the regions of image pixels and reduces the pixel size. The pixels of an image are extremely interrelated. Hence the special affiliation of neighbouring pixel is an abstract and significant distinctive of image segmentation.

The FCM clustering algorithm reduces the effect of noise.

The algorithmic steps are illustrated below 


\section{A HYBRID LEAF DISEASE DETECTION SCHEME USING GRAYCO-OCCURANCE MATRIX SUPPORT VECTOR MACHINE ALGORITHM}

Step 1: The images which are acquired from the camera have different pixel size.

Step 2: Resize the image and remove noise by median filter as per the equation (1).

$$
\operatorname{median}\{x(n)+y(n)\} \neq \operatorname{median}\{x(n)\}+\operatorname{median}\{y(n)\}
$$

Step 3: After resizing, standardize the segment and proceed towards pre-processing steps. This process is completely processed by HSV concept. Modification of image intensity or color map ethics is achieved to switch information losses aroused due to image resizing.

Step 4: Next, process and identify best optimum cluster value by FCM algorithm.

i. Consider $X=\left\{x_{1}, x_{2}, x_{3} \ldots, x_{n}\right\}$ as an information units with centroids $V=\left\{\mathrm{v}_{1}, \mathrm{v}_{2}, \mathrm{v}_{3} \ldots, \mathrm{v}_{\mathrm{c}}\right\}$.

ii. Initially, cluster centers 'c' is randomly distributed and processed with the fuzzy membership ' $\mu_{i j}$ '.

iii. The best known position of the pixel alongside the clusters discovered utilizing as a pioneer or the centroid esteem for FCM calculation.

Step 5: A lot of examples that is nearest to the ideal hyper plane is known as a Support vectorand it is responsible for determining the hyper plane. It can be represented as,

Hyper plane eq $(y)=$ weight vector $*$ support vectors + bias element

Step 6: The GLCM is made from a Gray scale picture. The GLCM is computes how frequently a pixel with dark worth I happens either on a level plane, happens either vertically, or corner to corner to adjoining pixels with the incentive with the value $\mathrm{j}$.

Step 7: The way toward gathering the pixels of each bunch with the centroid worth happens for almost $\mathrm{K}$ number of cycles,

Step 8: Output picture with the pixels of leaf sectioned and the infections affected area recognized is gotten from the proposed GCSVM calculation.

To obtain a GLCM technique, utilize the Gray co-matrix task. The GLCM is considered by registering how as often as possible a pixel with the power (dim level) regard 'I' occurs in a specific spatial relationship to a pixel with the value $\mathrm{j}$.

As per usual, the spatial relationship is described as the pixel of interest and the pixel to its snappy ideal (on a level plane neighboring), anyway you can demonstrate other spatial associations between the two pixels. Each segment $(i, j)$ in the resultant GLCM is only the aggregate of the events that the pixel with worth 'I' occurred in the predefined spatial relationship to a pixel with worth $\mathrm{j}$ in the data picture.

Since the planning required to process a GLCM for the full amazing extent of an image is prohibitive, graycomatrix scales the information picture. Normally, grayco-matrix uses scaling to diminish the amount of intensity regards in gray scale picture from 256 to eight.

The amount of dull measurements chooses the size of the GLCM. To control the amount of diminish measurements in the GLCM and the scaling of intensity regards, using the Num Levels and the Gray Limits parameters of the gray comatrix work. See the gray co-matrix reference page for more information. The GLCM can reveal certain properties about the spatial course of the diminish measurements in the surface picture. For example, if the larger pieces of the areas in the GLCM are thought along the corner to corner, the surface is coarse with respect to the predefined balance. You can in like manner get a couple of real measures from the GLCM. See Deriving Statistics from a GLCM for more information.

The principle thought of SVM is to guide preparing tests from info space into a higher dimensional element space by methods for a bit capacity, and after that locate the ideal isolating hyperplane that augments the edge between the two classes in the component space. Bolster Vector Machines (SVM) is the best grouping calculation regarding prescient exactness.

The SVM calculation has been created as far as arrangement or forecast technique between the factual and the AI calculations.

The primary point of SVM is to build up a hyperplane with most extreme edge can be figured as an enhancement issue. It depends on certain hazard limits of measurable learning hypothesis (for example basic hazard minimization). It can play out a non-direct arrangement calculation by utilizing the bit trap, certainly mapping their contributions to high-dimensional element spaces. The part trap permits building the classifier without unequivocally realizing the element space. SVMs discover a hyper plane with a most extreme separation to the nearest purpose of the two classes named as ideal hyper plane.

For an occurrence, given a lot of focuses a SVM finds a hyperplane with same class and same plane with biggest conceivable division of focuses which isolates the hyperplane named as Optimal Separating Hyperplane (OSH) which augments the separation between the two parallel hyper planes and can limit the danger of misclassifying instances of the test dataset.

\section{RESULT AND DISCUSSION}

To analyse the portrayal of the model, accurateness, precision and recall were used for evaluating classification results and mean squared error (MSE) and $R 2$ score were used for evaluating regression results.

The hybrid GLCM with SVM was chosen to solve this problem. Here, $\mathrm{X}=\left(\mathrm{x}_{1}, \mathrm{x}_{2} \ldots \mathrm{x}_{\mathrm{n}}\right)$ represents an image with ' $\mathrm{N}$ ' denotes number of pixels and it is separated in to ' $\mathrm{C}$ ' clusters, and ' $\mathrm{X}_{\mathrm{i}}$ ' denotes sorts of facts. The process is repeated for various iterative and verified. It reduces the overall cost function, which is represented as shown in the Equ (3).

$$
\mathrm{K}=\sum_{i=1}^{N} \sum_{i=1}^{C} b_{i j}{ }^{m}\left\|x_{g}-v_{i}\right\|^{2}
$$

Where $b_{i j}$ is the affiliation of pixel $x_{j}$ in the $i^{\text {th }}$ cluster center, $\mathrm{Vi}$ is the vertex of cluster canter. $\|$.$\| is a matrix and \mathrm{m}$ is a constant say $\mathrm{c}$.

The constant ' $\mathrm{m}$ ' decides the fuzziness of the result of each barrier.

In the FCM calculation the probability is legitimately reliant on the separation between the pixel and individual focus. 
Table 1: for Leaf Diseases FCM Iteration Grey Image Position Difference Values

\begin{tabular}{|c|c|c|c|c|c|c|c|c|c|c|c|c|c|}
\hline Iteration NO & \multicolumn{6}{|c|}{ Gray DiffNorm value } & \multicolumn{7}{|c|}{ PosiDiffNorm value } \\
\hline Iteration 1 & 1 & 4 & 9 & 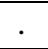 & 55 & 8 & 0 & & & 0 & & & \\
\hline on 2 & & & & & 2 & & & 1 & 7 & 5 & 6 & & 5 \\
\hline n 3 & 3 & & & & 8 & 2 & & 1 & 7 & & & & 9 \\
\hline$n$ & 2 & & & 6 & 2 & 4 & 5 & 1 & 7 & & & 6 & 0 \\
\hline n 5 & & & & 0 & 5 & 1 & 3 & 6 & 2 & & & & 0 \\
\hline n 6 & & & & 2 & 1 & 9 & 2 & 3 & 8 & & & 2 & 0 \\
\hline n 7 & 2 & & & 2 & 0 & 9 & 3 & 5 & & & & & 3 \\
\hline n 8 & & & & 9 & 5 & 0 & 6 & 5 & & & & & 5 \\
\hline on 9 & 2 & & & 5 & 3 & 2 & 4 & 7 & & & & & 2 \\
\hline on 10 & 2 & & & 3 & 8 & 6 & 4 & 3 & & 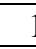 & & & 8 \\
\hline
\end{tabular}

From the table 1, ten iteration based on the Fuzzy $\mathrm{C}$ Means algorithm gray differential normal and positive normal values are listed values based on the differentiate after and before evaluated vales. Finally, 10iterationwere completed on the 5.57 seconds. From Table 1, it is observed that, all the parameters obtained in the evaluation test are in the acceptable limit. The quality of the image is stay tuned even after compression.

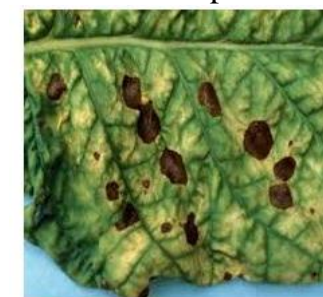

(

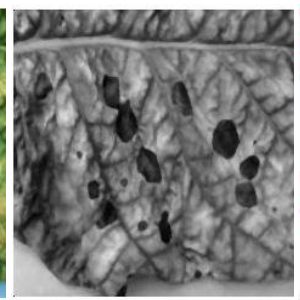

b

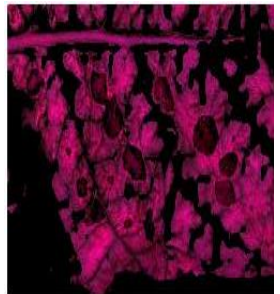

c )
Figure 4: (a) Input Sample, (b) Filtered Image,

(c) RGB concentrated sample

Each pixels in the delegated hue territory are set apart as closer view which are appeared white and different pixels are set apart as foundation are appeared dark.

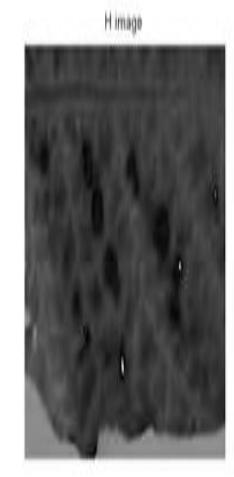

(a)

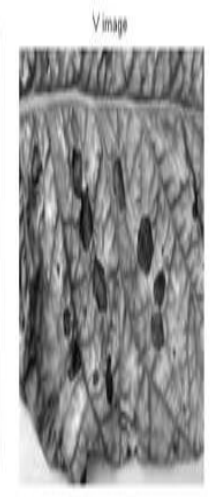

(b)

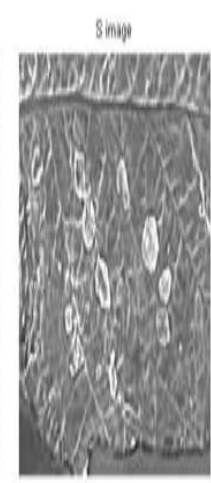

(c)

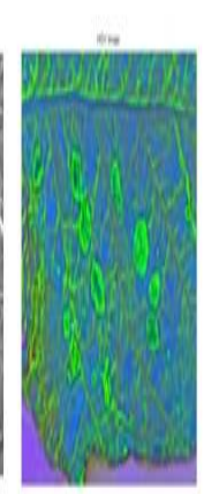

(d)
Figure 5: Morphological HSV pre-processing, (a) H processed, (b) V processed(c) S processed (d) HSV Image

The following figure 5 explains how HSV pre-processing is done in a captured image. Figure explains the HSV segmentation results of the input leaf image.

The diseases region find as a) hue image b) Saturation image c) vector image separated and final image for exact final merged HSV image can be viewed.

Partial segmentation of regions is done by the proposed HSV technique.

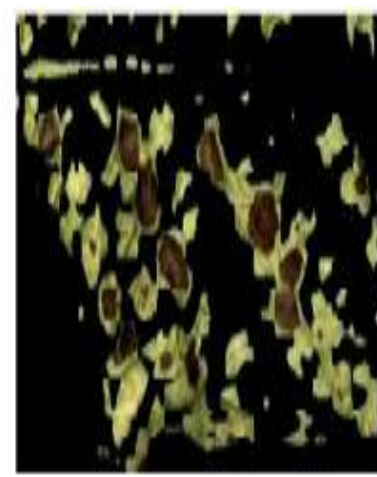

(a)

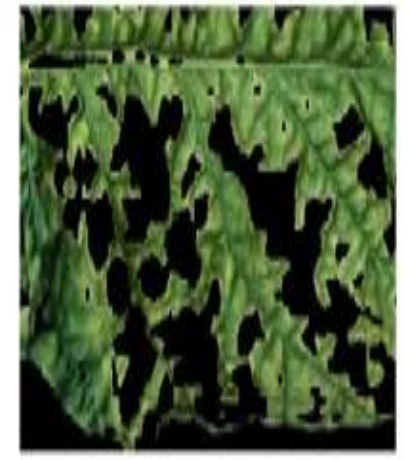

(b)
Figure 6: Fuzzy C-means clustering a) Iteration 1 and Iteration 2

Figure 6 describes about the Fuzzy $\mathrm{C}$ means clustering with various iterations. Fuzzy $\mathrm{C}$ means iterations are the whole images accomplished from the acquired leaves of clusters defined values. Moreover, no clear examination can be done with the input images. The previous methodologies are over whelmed with the help of the submitted hybrid GLCM embedded with SVM algorithm and that is verified successfully. Therefore, difficult instances of Diseases distinguishing proof and pixel division have been settled with the guide of the suggested hybrid calculation.

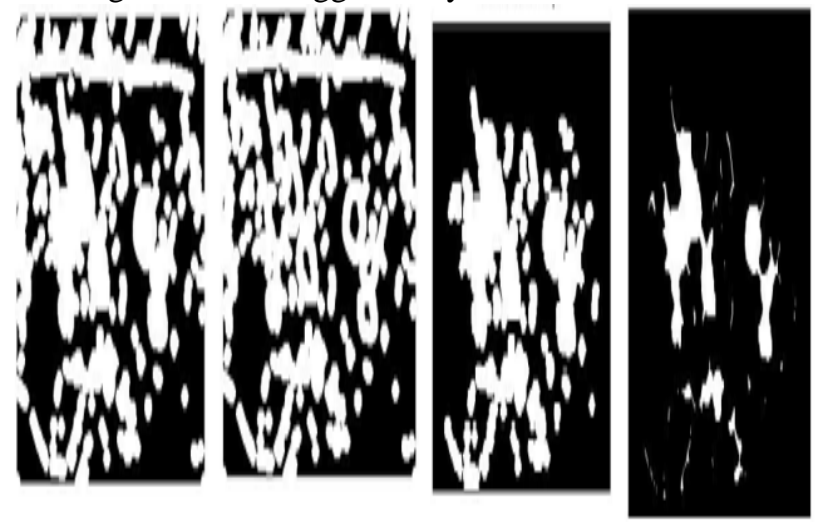

Figure 7: Segmentation process

(a)Binary Image, (b)Dilated gradient, (c) Cleared border, (d) Segmented image

Form the experimental results, various iterations are carried out. Figure 7 (a) leaf images with Contrast Enhancement - Figure 7(b) result the diagnosed the effected region using dilated method.

The above figure demonstrates the adequacy of the proposed strategy in settling testing horticultural datasets, where computer aided diagnosis is broadly favoured by the cures. Segregation between affected district and unaffected bits is made accessible in the after-effect of Figure 7(c). Inappropriate division of pixels areas is likewise perceptible.

This decreases the tally of genuine negative worth. Immaculate horticultural infections distinguishing proof and tissue division can be found in the outcome part of depicted table 2 division utilitarian qualities. Accurate distinguishing proof of ailments and division of tissue areas are portrayed by the proposed calculation and they are confirmed in the outcome part of Figure 8 (a) and (b).

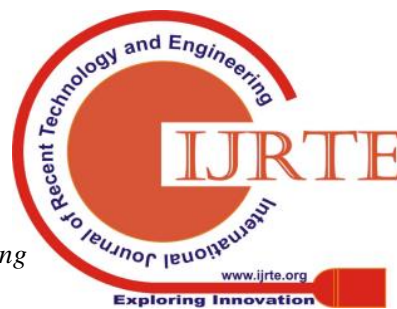



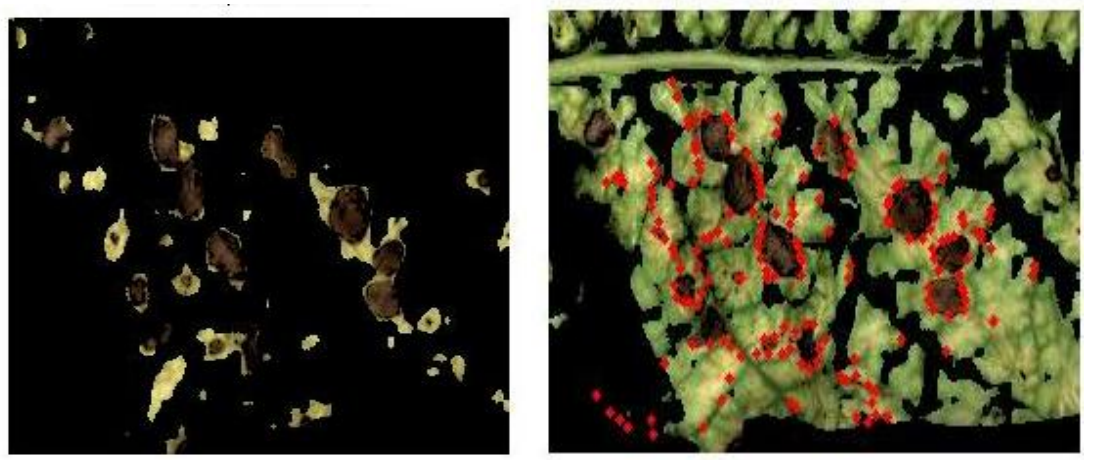

Figure 8: a) Segmentation defective plant detection b) Maximum diseases affected areas

Table2: Segmentation Function Evaluation and Values

\begin{tabular}{|c|c|c|}
\hline \multirow{2}{*}{$\begin{array}{l}\text { S.NO } \\
1\end{array}$} & FUNCTION EVALUATION & V A \\
\hline & Function evaluation $\quad 0$; & Value $2.824220 \mathrm{e}+06$ \\
\hline 2 & Function evaluation & Value $1.689169 \mathrm{e}+04$ \\
\hline 3 & Function evaluation & Value $4.672411 \mathrm{e}+02$ \\
\hline 4 & Function evaluation & Value $1.140523 \mathrm{e}+02$ \\
\hline 5 & Function evaluation & Value $9.668105 \mathrm{e}+01$ \\
\hline 6 & Function evaluation & Value $8.496362 \mathrm{e}+01$ \\
\hline 6 & Function evaluation & Value $8.495226 \mathrm{e}+01$ \\
\hline 7 & Function evaluation & Value $8.492776 \mathrm{e}+01$ \\
\hline 8 & Function evaluation & Value $8.492724 \mathrm{e}+01$ \\
\hline 1 & Function evaluation & Value $2.824220 \mathrm{e}+06$ \\
\hline 1 & Function evaluation & Value $5.588927 \mathrm{e}+03$ \\
\hline 1 & Function evaluation & Value $2.533837 \mathrm{e}+01$ \\
\hline 1 & tion evaluation & Value $2.208839 \mathrm{e}+01$ \\
\hline & Function evaluation & Value $2.207096 \mathrm{e}+01$ \\
\hline & Function evaluation & Value $8.202173 \mathrm{e}+00$ \\
\hline 1 & Function evaluation & Value $7.762325 \mathrm{e}+00$ \\
\hline 7 & Function evaluation & Value $7.760267 \mathrm{e}+00$ \\
\hline 8 & Function evaluation & Value $7.760166 \mathrm{e}+00$ \\
\hline 9 & Function evaluation & Value $7.528913 \mathrm{e}+00$ \\
\hline & Function evaluation & Value $7.426151 \mathrm{e}+00$ \\
\hline 1 & Function evaluation & Value $7.421383 \mathrm{e}+00$ \\
\hline & Function evaluation & Value $7.420761 \mathrm{e}+00$ \\
\hline & Function evaluation & Value $7.419066 \mathrm{e}+00$ \\
\hline 2 & Function evaluation & Value $7.419003 \mathrm{e}+00$ \\
\hline 2 & Function evaluation & Value $7.417207 \mathrm{e}+00$ \\
\hline 2 & Function evaluation & Value $7.417138 \mathrm{e}+00$ \\
\hline 2 & Function evaluation & Value $7.415693 \mathrm{e}+00$ \\
\hline 2 & Function evaluation & Value $7.415629 \mathrm{e}+00$ \\
\hline 9 & Function evaluation & Value $7.409095 \mathrm{e}+00$ \\
\hline 0 & Function evaluation & Value $7.409025 \mathrm{e}+00$ \\
\hline 1 & Function evaluation & Value $7.402263 \mathrm{e}+00$ \\
\hline 2 & Function evaluation & Value $7.402135 \mathrm{e}+00$ \\
\hline
\end{tabular}

The proposed GC-SVM model has been tested with captured image and it's been evaluated with accuracy, precision, recall, and F1-score parameters, which is

Table 3: Performance analysis of KNN and proposed GCSVM

\begin{tabular}{|c|c|c|c|c|c|c|c|c|}
\hline Disease $\mathrm{Na}$ & \multicolumn{2}{|c|}{ A c c u r a c y } & \multicolumn{2}{|c|}{ Precis ion } & \multicolumn{2}{|c|}{$\begin{array}{lllllll}R & e & c & a & l & l\end{array}$} & \multicolumn{2}{|c|}{ 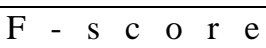 } \\
\hline & K N N & GCSVM & KNN & GCSVM & KNN & GCSVM & K N N & GCSVM \\
\hline & & & & & & & & \\
\hline & 130 & 07 & & 85 & 89 & 393 & & \\
\hline$L$ e a f & 0.9730 & 0.9523 & 1.00 & & 0.800 & 0.812 & 0.889 & 0.895 \\
\hline
\end{tabular}

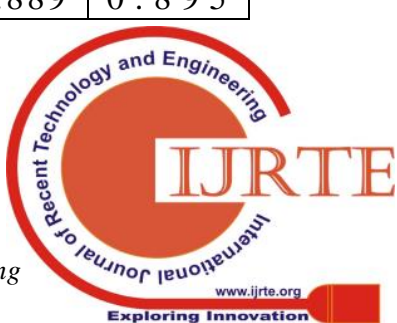




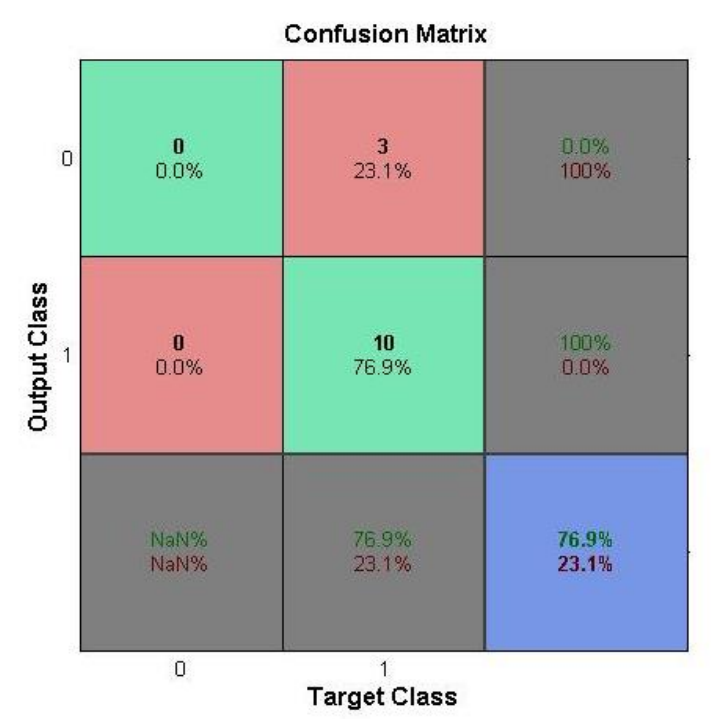

Figure 9: Leaf Diseases Detection Confusion Matrix

The disarray matrix in Fig. 9 demonstrates the quantity of concealed green part speaks to the accurately arranged disease among the complete number of pictures of each class. The parameters have been determined by considering the one individual ailment class as evident class and every different class are false class. A farmer can have a clear knowledge about the diseases and its location in the leaves. The value of Mean Square Error and Peak Signal to Noise Ratio obtained proves that there is minimum dissimilarity between the segmented images. To analyse the proposed model, accuracy, precision and recall were used for evaluating classification results and mean squared error (MSE) and $R 2$ score were used for evaluating regression results. The hybrid GLCM with FCMEANS was chosen to solve this problem.

Table 4: False Positive and negative \begin{tabular}{|l|l|l|}
\hline S u b j e c t $\mathbf{s}$ & Projected positive & Projected Negative \\
\hline
\end{tabular} \begin{tabular}{|l|l|l|}
\hline Actual positive & True Positive (TP) & False Negatives (FN) \\
\hline
\end{tabular} \begin{tabular}{|l|l|l|}
\hline Actual negative & False positives (FB) & True Negatives (TN) \\
\hline
\end{tabular}

Both sensitivity and FROC uses the terms in the confusion matrix. Two independent result sets were requested for the same test data using each of these evaluation measures.

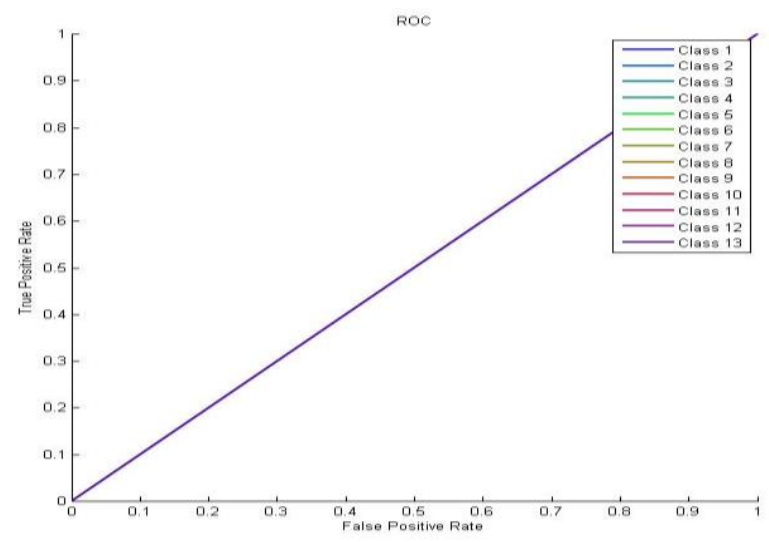

Figure 10: Gaussian regression diseases classification ROC class diagram

From the obtained values, it is concluded that the projected method has better option for disease detection in tea leaves. right and false ordered disease. The corner to corner

The GCSVM has the accuracy of $98 \%$ and precision of 1 . Since, this prototype module tested with various tea leaves collected from different regions. In future, this may help researchers to develop their efficient outcome.

\section{CONCLUSION}

The method reported in the article can be used to frame automatic leaf detection module for the early detection of plant infection. Through the effort, we have attempted to feature the issues related with the tea leaves and reasons for low yield loss in the developing nations like India.

Exactly, the tea leaves samples are collected from Udhagamandalam, Tamilnadu. Initially, various research methodologies are analysed and reviewed.

From that, specific median filters are considered for image noise removal. Later, the median filter is deployed for pre-processing the sample. Later, Fuzzy C Means clustering is utilized; the image is segmented with greater accuracy. Finally, the hybrid classifier method named as Gray-Level Co-Occurrence Matrix support vector machine is considered.

The performances of conventional methods are found to be limited for agricultural leaf and image identification. The performance of this hybrid model for compression process is improved by means image diseases detection is found to be better when compared to conventional compression methods and achieved higher accuracy $98 \%$.

\section{REFERENCES}

1. Kumar, S., Sharma, B., Sharma, V. K., Sharma, H., \&Bansal, J. C. (2018). Plant leaf disease identification using exponential spider monkey optimization. Sustainable Computing: Informatics and Systems.

2. Hossain, E., Hossain, M. F., \&Rahaman, M. A. (2019, February). A Color and Texture Based Approach for the Detection and Classification of Plant Leaf Disease Using KNN Classifier. In 2019 International Conference on Electrical, Computer and Communication Engineering (ECCE) (pp. 1-6). IEEE.

3. Chaudhary, P., Chaudhari, A. K., Cheeran, A. N., \&Godara, S. (2012). Color transform based approach for disease spot detection on plant leaf. International Journal of Computer Science and Telecommunications, 3(6), 65-70.

4. Sladojevic, S., Arsenovic, M., Anderla, A., Culibrk, D. \&Stefanovic, D. (2016). Deep neural networks based recognition of plant diseases by leaf image classification. Computational intelligence and neuroscience, 2016.

5. Harini, D. N. D., \&Bhaskari, D. L. (2011). Identification of Leaf diseases in Tomato Plant based on Wavelets and PCA. In 2011 World Congress on Information and Communication Technologies (pp. 978-1).

6. Rastogi, A., Arora, R., \& Sharma, S. (2015, February) Leaf disease detection and grading using computer vision technology \& fuzzy logic. In 2015 2nd international conference on signal processing and integrated networks (SPIN) (pp. 500-505).IEEE.

7. Al Bashish, D., Braik, M., \&Bani-Ahmad, S. (2011). Detection and classification of leaf diseases using K- 
means-based segmentation and. Information Technology Journal, 10(2), 267-275.

8. Patil, J. K., \& Kumar, R. (2011). Advances in image processing for detection of plant diseases. Journal of Advanced Bioinformatics Applications and Research, 2(2), 135-141.

9. Mix, C., Picó, F. X., \&Ouborg, N. J. (2003). A comparison of stereomicroscope and image analysis for quantifying fruit traits. Seed Technology, 12-19.

10. Abdullah, A., \&Umer, M. (2004). Applications of remote sensing in pest scouting: evaluating options and exploring possibilities. In Proceedings of the 7 th International Conference on Precision Agriculture and Other Precision Resources Management, Hyatt Regency, Minneapolis, MN, USA. St. Paul, MN: Precision Agriculture Center, University of Minnesota (pp. 1-13).

11. Ei-Helly, M., Rafea, A., Ei-Gamal, S., \&Whab, R. A. E. (2004). Integrating diagnostic expert system with image processing via loosely coupled technique. Central Laboratory for Agricultural Expert System (CLAES).

12. Tzionas, P., Papadakis, S. E., \&Manolakis, D. (2005, October). Plant leaves classification based on morphological features and a fuzzy surface selection technique. In Fifth international conference on technology and automation, Thessaloniki, Greece (pp. 365-370).

13. Du, J. X., Huang, D. S., Wang, X. F., \&Gu, X. (2006). Computer-aided plant species identification (CAPSI) based on leaf shape matching technique. Transactions of the Institute of Measurement and Control, 28(3), 275285.

14. Hu, R., Jia, W., Ling, H., \& Huang, D. (2012). Multiscale distance matrix for fast plant leaf recognition. IEEE transactions on image processing, 21(11), 4667-4672.

15. Prasad, S., Kudiri, K. M., \&Tripathi, R. C. (2011, February). Relative sub-image based features for leaf recognition using support vector machine. In Proceedings of the 2011 International Conference on Communication, Computing \& Security (pp. 343-346). ACM.

16. Ehsanirad, A., \& Kumar, S. (2010). Leaf recognition for plant classification using GLCM and PCA methods. Oriental journal of Computer Science \& technology, 3(1), 31-36.

17. Sladojevic, S., Arsenovic, M., Anderla, A., Culibrk, D., $\&$ Stefanovic, D. (2016). Deep neural networks based recognition of plant diseases by leaf image classification. Computational intelligence and neuroscience, 2016.

18. Wang, J., Zheng, P., Liu, H., \& Fang, L. (2016). Classification of Chinese tea leaves using laser-induced breakdown spectroscopy combined with the discriminant analysis method. Analytical Methods, 8(15), 3204-3209.

19. Hossain, M. S., Mou, R. M., Hasan, M. M., Chakraborty, S., \&Razzak, M. A. (2018, March). Recognition and detection of tea leaf's diseases using support vector machine. In 2018 IEEE 14th International Colloquium on Signal Processing \& Its Applications (CSPA) ( $\mathrm{pp}$. 150-154). IEEE.

20. Sun, Y., Jiang, Z., Zhang, L., Dong, W., \&Rao, Y. (2019). SLIC_SVM based leaf diseases saliency map extraction of tea plant. Computers and Electronics in Agriculture, 157, 102-109. 